\title{
The application of impedance measurement to assess biofilm development on technical materials used for water supply system construction
}

\author{
Mirela Wolf ${ }^{1, *}$, Teodora Traczewska $^{1}$, and Tomasz Grzebyk ${ }^{2}$
}

${ }^{1}$ Wroclaw University of Science and Technology, Faculty of Environmental Engineering, Wybrzeze Wyspiańskiego 27, 50-370 Wroclaw, Poland

${ }^{2}$ Faculty of Microsystem Electronics and Photonics, Wrocław University of Technology, Wybrzeze Wyspianskiego 27, 50-370 Wrocław, Poland

\begin{abstract}
The lack of biological stability of water which is introduced into the network, leads primarily to its secondary contamination during transport to the consumer. The water that is biologically unstable creates ideal conditions for colonization of the inner surface of pipelines by microorganisms and adhesion of their products (biocorrosion). The studies was conducted using the identified microorganisms isolated from the water supply network which accounted inocula in continuous culture of biofilm in CDC reactor. As a result of studies it was revealed the presence of biofilm formed on different materials polyethylene, polypropylene, polyvinyl chloride, polybutylene. Microbiological biodiversity of organisms inhabiting a biofilm of the diversity of nucleic acids was used. It was observed the amount of the psychrophilic bacteria oscillation in the effluent from the reactor. It was also determined the affinity of various bacteria to the plastic through adhesion measurement using impedance spectroscopy. For impedance measurements apparatus SIGNAL RECOVERY 7280 DSP LOCK-IN AMPLIFIER was used, recording impedance components (real and imaginary). The results will allow for the creation of biosensor systems that can be used in predicting health risks in connection with drinking water and taking corrective actions.
\end{abstract}

\section{Introduction}

The main task of the drinking water treatment plants is to maintain its proper purity and stability during transport to the consumer. Ensuring both chemical and biological stability of transported water is one of the key problems related to water quality management. Water quality may be reduced mainly by corrosion of pipes and water supply equipment. Corrosion is an extremely complex process, depending on the quality of the distributed water, hydraulic conditions, the material forming the network, and the presence of

*Corresponding author: mirela.wolf@pwr.edu.pl 
microorganisms. Biological corrosion is associated with degradation of material due to the activity of microorganisms, mainly of those remaining in close contact with the surface of the pipeline, forming the biofilm [1-3].

Mature biofilm is a complex structure comparable to the ecosystem. Microorganisms are suspended in the extracellular polymers, and the exchange of matter and genes occurs through an extensive system of canals. Beside the possibility of detachement and transfer of portions of a microbial biofilm to other parts of the network, further problem is the possibility of long-term survival or proliferation of pathogens (e.g. Legionella sp.) in the structures of the biofilm, among others, because of the limited penetration of disinfectants [4-6].

Traditional methods of assessing the microbiological quality of water are usually just the first step of identifying microorganisms, which directs further analyses using molecular biology methods. Standard methods do not reflect the actual species composition, because they are targeted at the bacteria that can be isolated and cultured in the laboratory. It is believed that cultivation techniques are able to obtain from $1-10 \%$ of the total bacteria present in the environment [7]. These methods are also tedious and labor intensive. They also require the presence of qualified staff and special care during the sample preparation, whereas the results of those tests are available after a few days [8]. Therefore, an attempt was made to develop methods for a quick analysis that would yield results in real time without having to prepare special additional samples. The proposed process for the continuous measurement will allow in the future for a constant monitoring of stages of the formation of biofilms, which will allow for control over the current risks to the consumer and to take appropriate preventive measures [9].

The article is aimed at explaining the problem that is the formation of biological growth on the surface of water pipes, despite introducing new technical materials. For this reason, the impedance sensor was used, which was colonized by the microorganisms during water flow. The measured impedance value provide a real-time information on the biofilm development.

\section{Materials and methods}

For the culture of the biofilm we have used a CDC reactor from Biosurface Technologies company (Fig 1A).The contents of the reactor was a synthetic tap water inoculated with a mixture of 11 strains from our own collection [10]. Three segments of pipes made of plastic (polyethylene, polypropylene, polyvinyl chloride, polybutylene) (Figure 1B) and a measuring sensor (Figure 1C) were placed in the reactor. After 14 days of pre-incubation in static conditions, the culture was carried out in flow conditions with constant stirring (100 rpm stirring) at room temperature. The flow rate of water in the system was similar to the actual value and was set at $0.3 \mathrm{~m} / \mathrm{s}$. In the work has been used a specially designed conductometric cell created with the procedure shown on Figure 2B. 


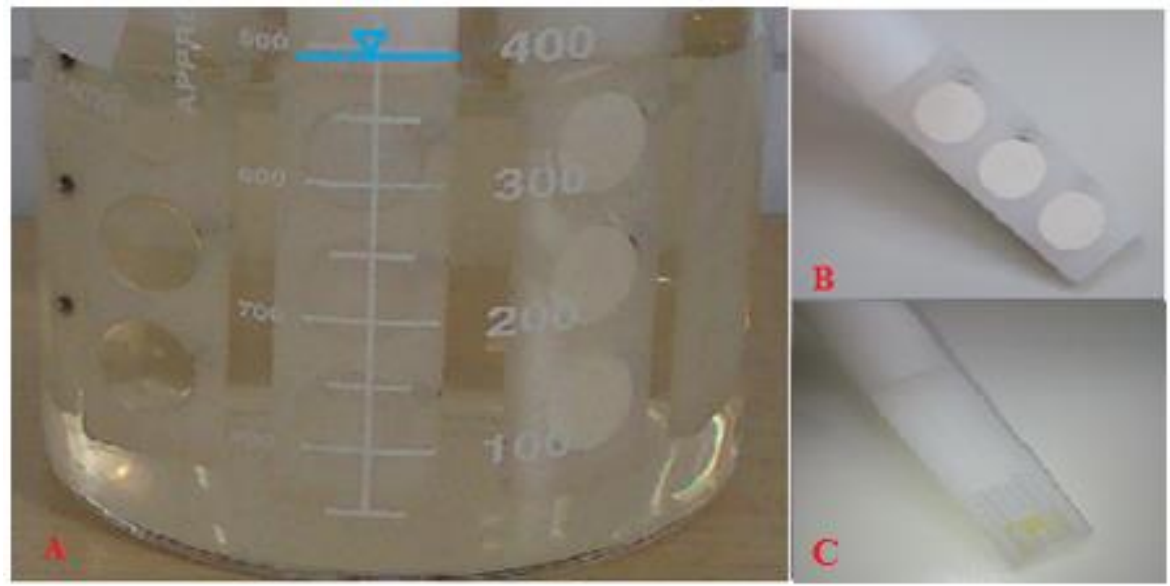

Fig. 1. Reactor with working fluid (A), handle to the technical materials (B-polyethylene), sensor attached to a connector (C).

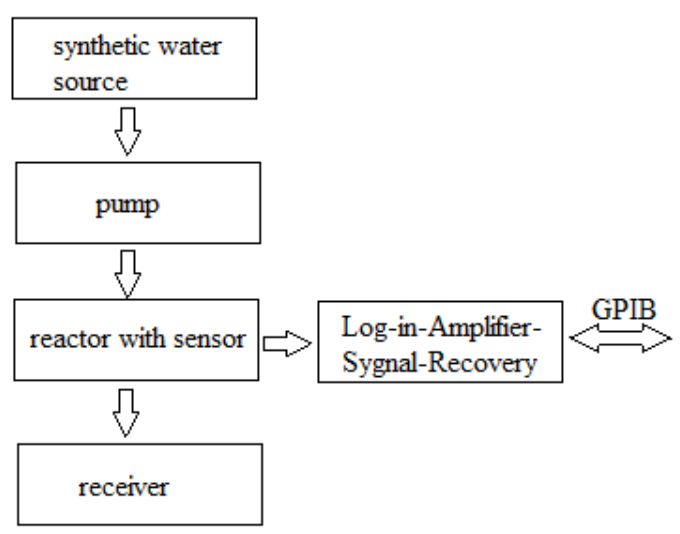

A

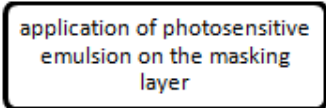

application on the base of the mask and its UV radiation

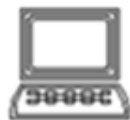

polimerisation of mask in the UV radiated places

cleansing of emulsion that has not polimerised (washing)

digestion of silicon dioxide in hydrofluoric acid (exposure of the conductive layers)

B

Fig. 2. Diagram of the measuring system-A and the process of fabrication of an impedance sensor using photolithography on ITO medium-B $[11,12]$.

The adhesion of bacterial cells to the sensor disturbed the current flow between electrodes, what resulted in the change of impedance value. The measurement was carried out using measuring equipment of Signal Recovery 7280 DSP Lock-in-amplifier. At the same time, along the measurement, the bacteria in the outflow of the reactor were quantified daily for 30 days. Using deep inoculation, $1 \mathrm{~cm}^{3}$ from dilution was applied to the medium with R2A agar. After $72 \mathrm{~h}$ of incubation at $20 \pm 2{ }^{\circ} \mathrm{C}$ grown colonies were counted. 
Biological growth on test materials and the sensor was monitored using a fluorescent dye DAPI (aqueous solution of 4,6-diamidino-2-phenyl-indol) and using a fluorescence microscope Olympus CX41. DAPI by binding to double-stranded DNA (dsDNA) present in the bacteria forms under the UV light $\lambda=365 \mathrm{~nm}$ is providing a highly fluorescent blue complex. For this purpose, $1 \mu \mathrm{l}$ of dye was applied onto the test material and then it was incubated in the dark for 20 minutes, then the samples were viewed under a microscope. Live bacterial cells dyed with DAPI are fluorescent blue [13].

An important element was to correlate the changes in impedance with the build-up of the biofilm created by the selected strains, therefore, in the first stage it was necessary to determine the frequency of measurements.

\section{Results and discussion}

The first attempt was made to determine the most appropriate frequency of the conductometric measurements. For this purpose, a sensor was placed in a homogeneous mixture of 11 strains in synthetic water. Figure 3 presents, as an example, the results of relative impedance for the frequencies from 1 to $100000 \mathrm{~Hz}$. The measurements showed that the frequency has no significant impact on the received signal, as the changes reached about $1 \%$, and the frequencies for which minimum and maximum values occurred did not overlap. The inoculum in a reactor was a mixture of 11 strains, each of which may be received in different ranges. It was impossible to explicitly select, either during the weekly or the daily measurement, for which frequency the changes were the most significant. Therefore, for further measurements the frequency was set at $100 \mathrm{~Hz}$, selected based on the literature [14].

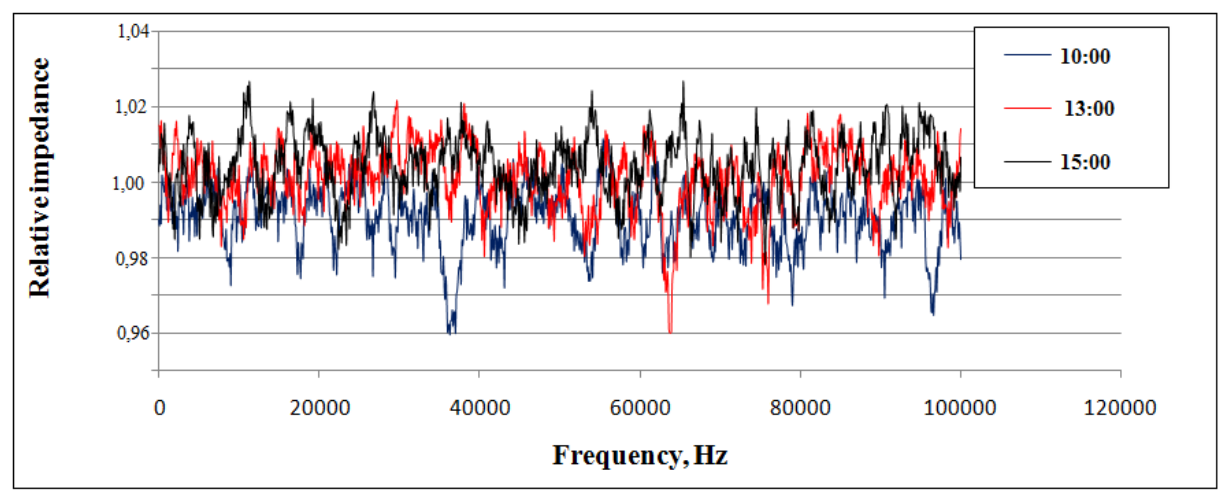

Fig. 3. Relative impedance measurement according to changing frequency.

Subsequently, the measurement of biofilm was carried out continuously in flow conditions for 30 days [15]. The measurements were carried out at intervals of 10 seconds. After the minimal fluctuations in the range from 0.650 to 0.725 in the first 5 days of the experiment, the value of the measured impedance was maintained for 20 days at approx. 0.670. After that time, a systematic increase in the observed impedance value up to 0.850 for the thirtieth day was observed (Fig. 4). The conditions of the experiment reflected the actual system of the water supply network, where the number of bacteria is small and is a mixture of several strains, mainly of psychrophilic bacteria. 


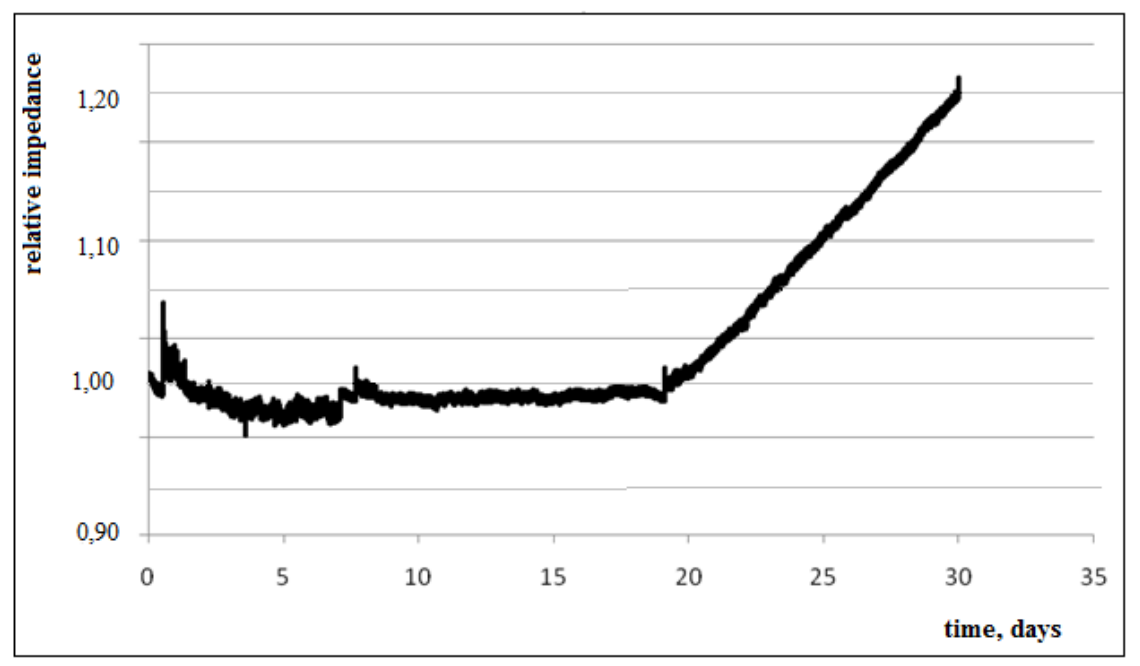

Fig. 4. Relative impedance during strains growth in synthetic water media.

Hadar Ben-Yoav, while measuring the impedance value in the range of $400 \mathrm{kHz}$ to $100 \mathrm{mH}$, demonstrated that the results depend on the growth phase of biofilm formed by E. coli [16]. Similar observations were achieved by [17] in study which involved measurements of impedance before and after the adhesion of cells of bacteria Pseudomonas stutzeri on indium-tin-oxide (ITO) plate, for variable range of frequencies [17]. In both cases, a culture of a single strain has been used for study, and the duration of the experiment was a few days. Hadar Ben-Yoav have demonstrated that better effects are obtained for lower frequencies, hence the obtained results differ from those obtained by [17].

Due to potential partial leaching of the bacteria from the system, a quantitative analysis of the water flowing out of the reactor was carried out. This study indicated that the greatest amount of bacteria in the outflow was observed after the change of conditions as a result of the introduction of water flow (Figure 5).

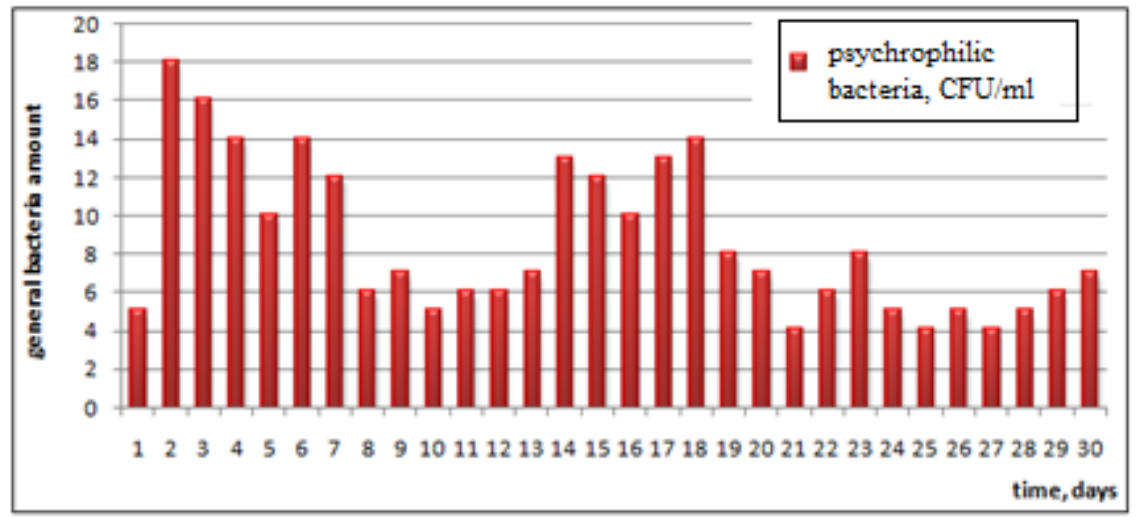

Fig. 5. Quantitative inoculation onto reasoner's $2 \mathrm{~A}$ agar (2A) of samples from the outflow from the biofilm reactor. 
Bacterial count declined steadily from $18 \mathrm{CFU} / \mathrm{ml}$ on the second day to about $6 \mathrm{CFU} / \mathrm{ml}$ from $8^{\text {th }}$ to $13^{\text {th }}$ day of the duration of the experiment. Between $14^{\text {th }}$ and $18^{\text {th }}$ day an increase to about $14 \mathrm{CFU} / \mathrm{ml}$ was observed, and another decrease of the number of microorganisms. From day $19^{\text {th }}$ to the end of the experiment there was a stabilization of the system and the flushed cells ranged from 4-6 CFU/ml. This suggests stabilization of the system as a result of the process completion of biofilm maturation.

To visualize all the bacteria colonizing the technical materials and the sensor, staining with a fluorescent dye was used (Fig. 6).
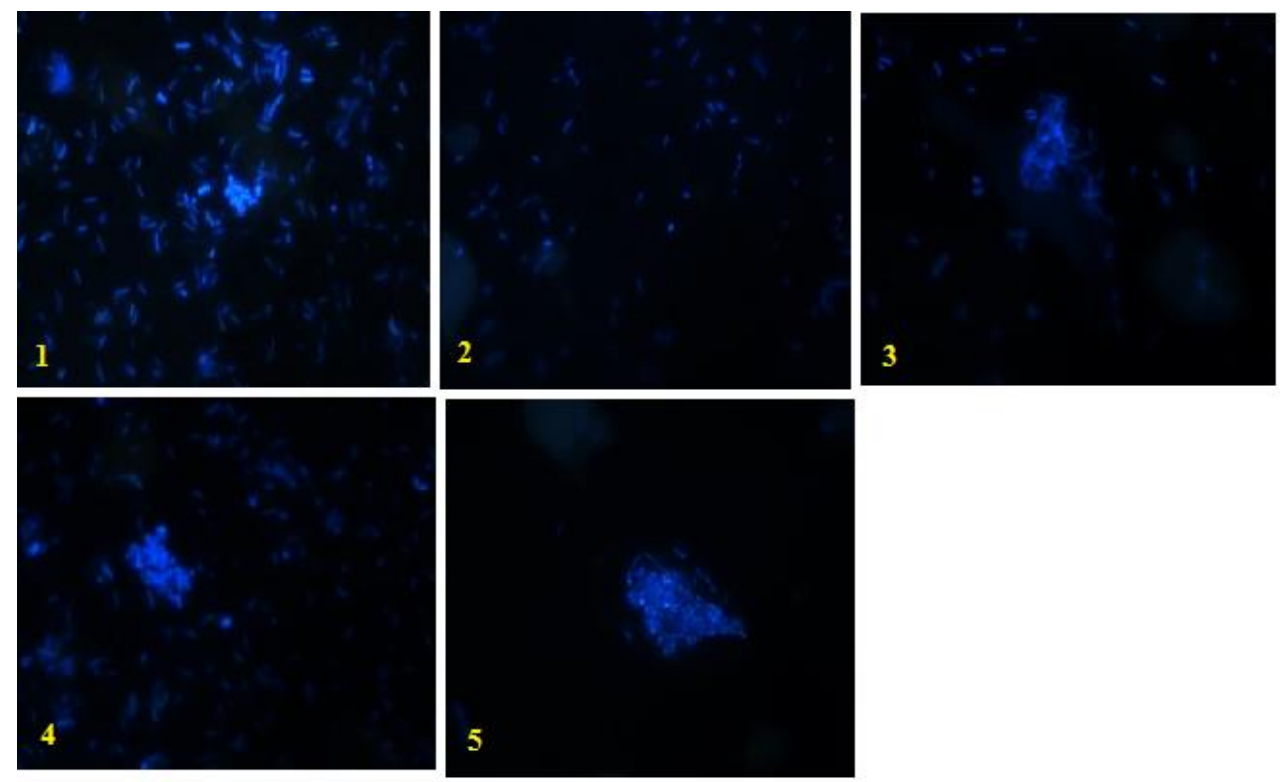

Fig. 6. The total amount of bacteria accumulated on parts of pipeline and sensor; 1 - polypropylene, 2 - polyethylene, 3 - polyvinyl chloride, 4 - polybutylene, 5 - sensor, seen in a fluorescent microscope under 1000x immersion magnification.

The most numerously colonized plastic was the polypropylene (6.1). In turn, the images obtained for polyvinyl chloride (6.3) and polybutylene (6.4) were very similar to those of the sensor (6.5). The least colonized was the area of polyethylene (6.2).

The study confirmed the results of Wang demonstrating that the material from which the water supply network is made of can have a significant effect on the amount, frequency and type of biofilm formation, the presence of pathogens as well as the effectiveness of used disinfectants [18].

The results of studies on the susceptibility to biological growth of technical materials used to build water supply networks show significant discrepancies. Niquettei demonstrated that the materials from synthetic materials (PE, PVC) were less colonized by biofilm than pipes made of iron, copper and steel, however, on the other hand, Zacheusi demonstrated a lack of significant differences in colonization of synthetic materials (PE, PVC) and steel $[19,20]$. Schwartz have shown in their work that more bacterial cells are gathered on the surface of polyethylene, what is favorable for a faster growth of biofilm than on the surface of PVC [21]. Not with standing, according to Ginige the most frequently selected material for the water supply network is high density polyethylene, due to its low cost of production and durability [22]. 


\section{Conclusion}

It is necessary to select the appropriate material for construction of the water supply network to prevent bacterial growth in distribution systems for drinking water. Corrosion, deteriorating water quality, taste and smell as well as increased numbers of pathogens are just some of the negative effects caused by the presence of biofilm. The total elimination of biofilm from the network seems unlikely, even if the amount of absorbable organic carbon will be reduced to $0.2 \mathrm{mgC} / \mathrm{m}^{3}$ and it will be manageable to maintain an adequate level of disinfectant in the network. The basic cause is the material used to build the network and its properties. All technical materials have micro cracks on their surface, a result of the manufacturing process, which are susceptible to the adhesion of bacterial cells. Thus, sensors should be built on substrates with physical characteristics similar to the material used for the construction of water supply systems. The measurement of impedance can be correlated with the cell adhesion of bacteria to the surface of the water pipes and, after detailed studies, it could serve as a method for monitoring the dynamics of the biofilm development, providing a tool for microbiological quality control of tap water.

\section{Acknowledgments}

The research was finance by the funds of the Polish Ministry of Science and Higher Education for young scientist and doctoral students; order numer: 0402/0082/16.

\section{References}

1. M. Ginige, J. Wylie, J. Plumb, Biofouling 27, 2 (2011)

2. J. Rubulis, T. Juhna, Water Sci.Technol. 55, 8-9 (2007)

3. D. Berry, Xi Chuanwu, L. Raskin, Curr. Opin. Biotech. 17, 3 (2006)

4. H. Wang, C. Hu, X. Hu, M. Yang, J. Qu, Water Res. 46, 4 (2012)

5. J. Wingender, F. Hans-Curt, Int. J. Hyg. and Envir. Healh. 214, 6 (2011)

6. T. Battin, K. Besemer, M. Bengtsson, A. Romani, A. Packmann, Nat. Rev. Micrbiol. 14 (2016)

7. S. Nevel, S. Koetzsch, W. Hans-Ulrich, N. Boom, F. Hammes, J. Biol. Met. 94, 2 (2013)

8. Ch. Peng, G. Korshin, R. Valentine, A. Hill, M. Friedman, S. Reiber, Water Res. 44, 15 (2010)

9. M. Schock, R. Hyland, M. Welch, Envir. Sci. Tech. 42, 12 (2008)

10. I. Biedroń, M. Traczewska, G. Płaza, Ann. Set Envi. Pro. 18 (2016)

11. Z. Zimniak, M. Marciniak, I. Zubel, Scientific Works of Warsaw University of Technology, 267 (2015) (in Polish)

12. S. Mannsfeld, B. Tee, R. Stoltenberg, Ch. Chen, S. Barman, B. Muir, A. Sokolov, C. Reese, Z. Bao, Nat. Mater. 9 (2010)

13. A. Ferro, T. Mestre, P. Corneiro, I. Sahumbaiev, R. Seruca, J. Sanches, Lab. Invest. 6 (2017)

14. J. Parades, S. Becerro, S. Arana, Sen. Acu. Chem. 195 (2015)

15. S. Nevel, K. Roy, N. Boon, FEMS Microbiol. Ecol. 85 (2013) 
16. H. Ben-Yoav, A. Freeman, M. Sternheim, Y. Shacham-Diamand, Electrochim. Acta 56, 23 (2011)

17. S. Bayoudh, A. Othmane, L. Ponsonnet, H. Ben Ouada, Physicochem. Eng. Asp. 318 (2008)

18. H. Wang, S. Masters, M. Edwards, J. Falkinham, A. Pruden, Env. Sci. Tech. 48, 3 (2014)

19. P. Niquette, P. Servais P, R. Savoir, Water Res. 24, 6 (2000)

20. O. Zacheus, E. Iivanainen, T. Nissinen, M. Lehtola, P. Martikainen, Water Res. 34, 1 (2000)

21. T. Schwartz, S. Hoffmann, U. Obst, Water Res. 32, 9 (1998)

22. M. Gringe, S. Garbin, J. Wylie, C. Krishna, Plos One, 6 (2017) 